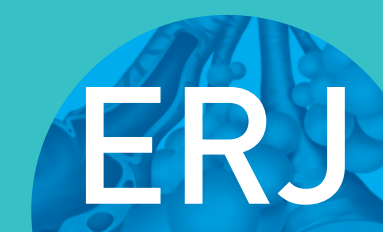

open research

\title{
Inhaled nitric oxide decreases pulmonary endothelial nitric oxide synthase expression and activity in normal newborn rat lungs
}

\author{
Thông Hua-Huy ${ }^{1}$, Sy Duong-Quy ${ }^{1}$, Hoa Pham², Julien Pansiot ${ }^{2}$, \\ Jean-Christophe Mercier ${ }^{3}$, Olivier Baud ${ }^{2,4}$ and Anh Tuan Dinh-Xuan ${ }^{1}$
}

Affiliations: ${ }^{1}$ Laboratoire de Physiologie respiratoire EA-2511, Université Paris Descartes, Service de Physiologie-Explorations fonctionnelles, Assistance Publique-Hôpitaux de Paris, Hôpital Cochin, Paris, France. ${ }^{2}$ INSERM, UMR1141, Université Paris Diderot, Paris, France. ${ }^{3}$ Service des Urgences Pédiatriques, Assistance Publique-Hôpitaux de Paris, Hôpital Robert-Debré, Paris, France. ${ }^{4}$ Réanimation et pédiatrie néonatales, Assistance Publique-Hôpitaux de Paris, Hôpital Robert-Debré, Paris, France.

Correspondence: Anh Tuan Dinh-Xuan, Service de Physiologie-Explorations Fonctionnelles, Hôpital Cochin, 27 rue du faubourg Saint-Jacques, 75014 Paris, France. E-mail: anh-tuan.dinh-xuandacch.aphp.fr

ABSTRACT Inhaled nitric oxide (iNO) is commonly used in the treatment of very ill pre-term newborns. Previous studies showed that exogenous NO could affect endothelial NO synthase (eNOS) activity and expression in vascular endothelial cell cultures or adult rat models, but this has never been fully described in newborn rat lungs. We therefore aimed to assess the effects of iNO on eNOS expression and activity in newborn rats.

Rat pups, post-natal day (P) 0 to $\mathrm{P} 7$, and their dams were placed in a chamber containing $\mathrm{NO}$ at $5 \mathrm{ppm}$ (iNO-5 ppm group) or $20 \mathrm{ppm}$ (iNO-20 ppm group), or in room air (control group). Rat pups were sacrificed at P7 and P14 for evaluation of lung eNOS expression and activity.

At P7, eNOS protein expression in total lung lysates, in bronchial and arterial sections, was significantly decreased in the iNO-20 ppm versus control group. At P14, eNOS expression was comparable among all three groups. The amounts of eNOS mRNA significantly differed at P7 between the iNO-20 ppm and control groups. NOS activity decreased in the iNO-20 ppm group at P7 and returned to normal levels at P14. There was an imbalance between superoxide dismutase and NOS activities in the iNO-20 ppm group at P7.

Inhalation of NO at $20 \mathrm{ppm}$ early after birth decreases eNOS gene transcription, protein expression and enzyme activity. This decrease might account for the rebound phenomenon observed in patients treated with iNO.

@ERSpublications

Inhaled NO decreases endogenous NO synthesis and favours rebound pulmonary hypertension after inhaled NO withdrawal http://ow.ly/WNDq2

Received: Aug 202015 | Accepted after revision: Dec 162015 | First published online: Month 02016

Conflict of interest: None declared.

The content of this work is @ the authors or their employers. Design and branding are @ERS 2016. This article is open access and distributed under the terms of the Creative Commons Attribution Non-Commercial Licence 4.0. 
Clinical signposts

- Inhaled nitric oxide (iNO) at 20 ppm for 1 week decreases endothelial NO synthase (eNOS) gene expression and enzyme activity in newborn rats.

- Reduced eNOS expression activity might account for rebound pulmonary hypertension after iNO withdrawal.

- Caution should therefore be exercised when weaning newborns with iNO.

- The inhibitory effects of iNO on eNOS expression and activity are transient, and the enzyme recovers its activity and expression 1 week after iNO withdrawal.

\section{Introduction}

Inhaled nitric oxide (iNO) is commonly used in the treatment of very ill pre-term newborns with high risks of persistent pulmonary hypertension and hypoxaemic respiratory failure [1]. NO is a selective pulmonary vasodilator, capable of reducing pulmonary vascular resistance and inhibiting vascular remodelling. In animal models, iNO has been found to promote angiogenesis, decrease apoptosis, and enhance surfactant function and alveolar formation $[1,2]$. Notwithstanding these theoretical beneficial effects, recent clinical trials found that iNO does not reduce morbidity of bronchopulmonary dysplasia in pre-term infants $[3,4]$. One of the most important side-effects when using iNO therapy for pulmonary hypertension is the acute increase of pulmonary arterial pressure after iNO withdrawal, known as rebound pulmonary hypertension $[5,6]$.

Several studies have been performed to identify the mechanisms of this phenomenon. Results from most of these studies showed a decrease in NO synthase (NOS) activity in lungs, accounting for rebound pulmonary hypertension in animal models [7-13]. It has been demonstrated that the inhibitory effects of iNO on NOS activity were associated with the induction of the endothelin (ET)-1/ET-A receptor pathway in newborn lambs [9-13] or with increased production of superoxide anion followed by a formation of peroxynitrite that in turn inactivates eNOS in pulmonary endothelial cells [7, 10]. However, studies investigating the effects of iNO on eNOS expression showed contradictory results, depending on the dose of iNO and experimental conditions [7-13].

In our previous study, iNO enhanced rat lung development at early post-natal life, probably through increased expression of haem oxygenase-1, vascular endothelial growth factor and matrix metallopeptidase-2 pathways in full-term rat pups [2]. The present study aimed to assess the effects of iNO at low dose $(5 \mathrm{ppm})$ or high dose $(20 \mathrm{ppm})$ on the activity and expression of eNOS in newborn rat lungs. We also evaluated the activity of superoxide dismutase (SOD), the main antioxidant enzyme in lung tissue of newborn rats treated with iNO.

\section{Materials and methods}

\section{Animal models}

All experimental protocols and animal housing procedures were approved by the National Institute of Health and Biomedical Research (INSERM, UMR1141, Paris, France), and complied with the institutional instructions for animal care and use. Timed-pregnant Sprague-Dawley rats were maintained at $20-24^{\circ} \mathrm{C}$ in a room with a 12-h light/dark cycle and free access to standard food. Controls for rat behaviours, food and drink sources, and gas concentrations $\left(\mathrm{NO}, \mathrm{NO}_{2}\right.$ and $\left.\mathrm{O}_{2}\right)$ were performed twice a day on weekdays and once a day on weekends.

\section{Experimental protocols}

All pregnant Sprague-Dawley rats were kept in normoxic conditions and randomly divided into three groups at the last day of their gestation: control (room air), low-dose iNO (iNO-5 ppm) and high-dose iNO (iNO-20 ppm) groups [14]. Rats from the iNO groups were placed in a transparent $50 \times 50 \times 70$-cm Plexiglas chamber that was connected to the NO source (BioSpherix, Redfield, IL, USA) so that rat pups would receive $\mathrm{NO}$ from their first inspiration. The concentration of $\mathrm{NO}_{2}$ was continuously monitored and kept at $<1 \mathrm{ppm}$ so that potential harmful effects of $\mathrm{NO}_{2}$, i.e. generation of methaemoglobin, could be prevented. The iNO period lasted for 7 days. Rat pups were sacrificed at post-natal day (P) 7 to study early lung development and at P14 to study late lung development. For the P14 group, all rats (control, iNO-5 ppm and iNO-20 ppm groups) were maintained under the same conditions with room air from P7 to P14.

\section{Tissue preparations}

Litter sizes were between 10 and 16; at least six littermates per group were killed by decapitation. The thoracic cavity was opened, and the lungs were removed and weighed. The left lung was washed in $0.9 \%$ $\mathrm{NaCl}$ ( $\mathrm{pH}$ 7.4), fixed with paraformaldehyde buffer (4\% in PBS, pH 7.4) until total submersion (2-5 days) 
and waxed in paraffin using an automated procedure (Shandon Citadel 2000 Tissue Processor; Rankin Biomedical, Holly, MI, USA). Lung blocks were cut into $5-\mu \mathrm{m}$ sections for immunohistochemical study. The right lung was snap-frozen in liquid nitrogen and stored at $-80^{\circ} \mathrm{C}$ until use for quantitative reverse transcriptase PCR (RT-PCR) or Western blot assays.

\section{Chemical reagents and antibodies}

All chemical reagents were purchased from Sigma-Aldrich (St Louis, MO, USA) except VECTASTAIN Elite ABC-Peroxidase kits (PK-7100) and VectaMount Permanent Mounting Medium (H-5000) for immunohistochemical staining, which were from Vector Laboratories (Burlingame, CA, USA). Primary antibodies included a rabbit anti-endothelial NOS (eNOS) antibody from BD Transduction Laboratories (San Jose, CA, USA; 610297) and a mouse anti- $\beta$-actin antibody from Santa Cruz Biotechnology (Dallas, TX, USA; sc-47778). Secondary antibodies were horseradish peroxidase (HRP)-conjugated goat anti-rabbit IgG (Santa Cruz Biotechnology; sc-2004) and goat anti-mouse IgG (Santa Cruz Biotechnology; sc-2005) antibodies for Western blotting, and biotinylated goat anti-rabbit IgG antibody (Vector Laboratories) for immunohistochemistry.

\section{Immunohistochemistry for eNOS}

Immunohistochemical staining for eNOS on rat lung sections was performed as previously described [15] with a minor modification. Sections of rat lung tissues were incubated with primary anti-eNOS antibody diluted to 1:50 (overnight, $4^{\circ} \mathrm{C}$ ). For semiquantitative analyses, eNOS expression was separately evaluated in bronchial and arterial sections, using arbitrary intensity scores, assigned as no staining (0), focal staining (1), diffuse weak staining (2), diffuse moderate staining (3) and diffuse strong staining (4). For each animal, at least 10 images ( $\times 400$ magnification) were randomly taken and "blindly" scored by two observers. Mean values of eNOS expression for each animal were used for comparison among groups of rat pups (control, iNO-5 ppm and iNO-20 ppm groups).

\section{Western blotting analysis for eNOS}

Protein extraction from frozen lung samples was performed as previously described [2]. For Western blots, equal amounts of pulmonary homogenates $(50 \mu \mathrm{g}$ per animal) were subjected to $7.5 \%$ SDS-PAGE and then transferred to polyvinylidene difluoride membranes (Immobilon-P; Millipore, Molsheim, France). Membranes were blocked in 5\% nonfat milk for $1 \mathrm{~h}$ at room temperature and then hybridised with primary anti-eNOS antibody $\left(1: 500\right.$, overnight, $\left.4^{\circ} \mathrm{C}\right)$. Blots were revealed after incubation with appropriate secondary HRP-conjugated antibody using enhanced chemiluminescence reagent (ECL Plus; Amersham, Courtaboeuf, France). Protein densities were quantified using the Genius2 image software system (Syngene, Cambridge, UK) and normalised to $\beta$-actin densities.

\section{Quantitative RT-PCR experiments}

Transcript levels of eNOS in lung tissues were determined by quantitative RT-PCR using a LightCycler ${ }^{\circ}$ 480 System (Roche Applied Biosystems, Foster City, CA, USA) and normalised to hypoxanthine-guanine phosphoribosyltransferase (HPRT) as previously described [16].

Oligonucleotide primers were designed with the Primer Express software package (Applied Biosystems, Saint Aubin, France) and purchased from Eurogentec (Liege, Belgium): eNOS forward: 5'-GGAGAAGATGCCAAGGCTGCTG-3'/eNOS reverse: 5'-CTTCCAGTGTCCAGACGCACCA-3' (224 bp) and HPRT forward: 5'-GGTGAAAAGGACCTCTCGAA-3'/HPRT reverse: 5'-CAAGGGCATATCCAACAACA-3' (260 bp).

Total RNA was extracted from frozen lung tissues using a Qiagen RNeasy Mini kit (Courtaboeuf, France). RNA quality and concentrations were assessed using a NanoDrop 1000 spectrophotometer (NanoDrop, Wilmington, DE, USA), based on relative absorbance at 260 versus $280 \mathrm{~nm}$. Total RNA ( $1 \mu \mathrm{g}$ per sample) was subjected to reverse transcription using an iScript cDNA Synthesis kit from Bio-Rad (Hercules, CA, USA).

For quantitative RT-PCR, samples were denatured at $95^{\circ} \mathrm{C}$ for $3 \mathrm{~min}$, followed by 40 cycles of $60 \mathrm{~s}\left(96^{\circ} \mathrm{C}\right.$ for $20 \mathrm{~s}$, $60^{\circ} \mathrm{C}$ for $20 \mathrm{~s}$ and $72^{\circ} \mathrm{C}$ for $20 \mathrm{~s}$ ), fusion at $68^{\circ} \mathrm{C}$ for $1 \mathrm{~min}$ and cooling at $45^{\circ} \mathrm{C}$ for $30 \mathrm{~s}$. Relative quantification values of the target genes were standardised according to the value of HPRT used as housekeeping gene.

\section{Determination of nitrate concentrations in rat lung homogenates}

A NOS activity assay kit was used to measure newly synthesised NO from L-arginine by the action of NOS in the presence of essential cofactors, according to the manufacturer's instructions (Cayman Chemical, Ann Arbor, MI, USA). The final products of the reaction were nitrates, measured by a colorimetric method $(540 \mathrm{~nm})$, which indirectly represented the activity of NOS. Nitrate concentrations were determined by extrapolating from a standard curve. 


\section{Analyses of SOD activity}

For analyses of SOD activity, frozen lung tissues were homogenised in ice-cold $0.1 \mathrm{M}$ Tris/HCl, $\mathrm{pH} 7.4$ containing $0.5 \%$ Triton X-100, $5 \mathrm{mM} \beta$-mercaptoethanol and $0.1 \mathrm{mg} \cdot \mathrm{mL}^{-1}$ phenylmethylsulfonyl fluoride. Tissue homogenates were centrifuged at $14000 \times \mathrm{g}$ for $10 \mathrm{~min}$ at $4^{\circ} \mathrm{C}$ and the supernatants were harvested. Total protein levels were measured using a bicinchoninic acid protein assay kit (Pierce Biotechnology, Rockford, IL, USA). The SOD activity was determined using a SOD activity assay kit (BioVision, Mountain View, CA, USA) according to the manufacturer's instructions. Briefly, this kit utilises the cell proliferation reagent WST-1 that produces a water-soluble formazan dye upon reduction with superoxide anion. The rate of the reduction with superoxide anion is linearly related to the xanthine oxidase activity and is inhibited by SOD. The inhibition activity of SOD can be determined by a colorimetric method. Relative SOD activity (inhibition rate (\%)) was normalised by protein amount of each sample.

\section{Statistical analysis}

Data are presented as mean \pm SEM. Statistical comparisons were performed with ANOVA with Tukey's post hoc test using the Prism 5 software (GraphPad, San Diego, CA, USA). Differences were considered significant when $\mathrm{p}<0.05$.

\section{Results}

There were no differences in lung-to-body weight and wet-to-dry lung weight ratios between the three groups of rat pups at P7 and P14 (data not shown).

\section{Decreased eNOS expression in the lungs from iNO-20 ppm rat pups}

Protein expression of eNOS was semiquantitatively evaluated by Western blot in rat lung homogenates. At P7, we observed a significant difference $(p=0.0008)$ in eNOS expression between the three groups of rat pups (figure 1a). Tukey's post hoc analyses showed that eNOS expression decreased in the iNO-20 ppm group to $41 \%$ of that of the control group $(\mathrm{p}<0.01)$, whilst there was no difference in eNOS protein between the iNO-5 ppm and control groups $(\mathrm{p}>0.05)$. At P14, eNOS expression was not different between the three groups of rat pups ( $\mathrm{p}=0.06)$ (figure $1 \mathrm{~b}$ ).

Immunohistochemical staining on paraffin-embedded rat lung sections showed a strong expression of eNOS in arterial endothelium and, to a lesser extent, in bronchial epithelium (figure 2). We assessed the intensity of eNOS expression separately in arterial and bronchial sections by an arbitrary scoring system as previously described [15]. At P7, eNOS expression was significantly different in arterial $(p=0.0006)$ and bronchial $(p=0.0003)$ structures between the three groups of rat pups (figure $3 \mathrm{a})$. There was a significant
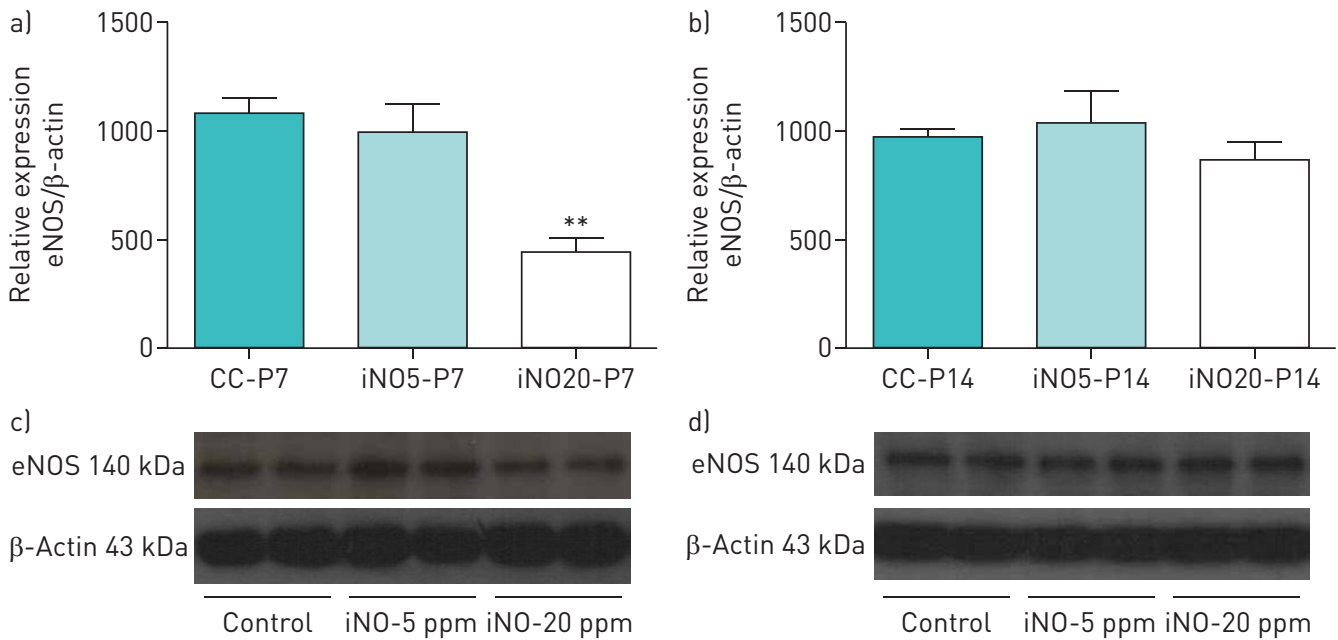

FIGURE 1 Expression of endothelial nitric oxide synthase (eNOS) assessed by Western blot in whole-lung homogenates. a, b) Densitometric values for relative eNOS protein (normalised to $\beta$-actin) at a) post-natal day (P) 7 and b) P14. CC: rat pups kept in room air (control group); iN05: rat pups kept in a Plexiglas chamber continuously filled with $\mathrm{NO}$ at $5 \mathrm{ppm}$ (iNO-5 ppm group); iNO20: rat pups kept in a Plexiglas chamber continuously filled with NO at 20 ppm (iNO-20 ppm group). c, d) Representative Western blots for whole-lung protein extracts at c) P7 and d) P14, separated on 7.5\% SDS-PAGE, electrophoretically transferred to polyvinylidene difluoride membranes and analysed with a specific antiserum raised again eNOS (upper bands) or $\beta$-actin (lower bands). At P7, expression of eNOS was significantly decreased in the iNO-20 ppm group as compared with that in the control group $(p<0.05)$. At $P 14$, there was no significant difference among the three groups of rats $(p>0.05)$. ${ }^{* *}: p<0.01$. 

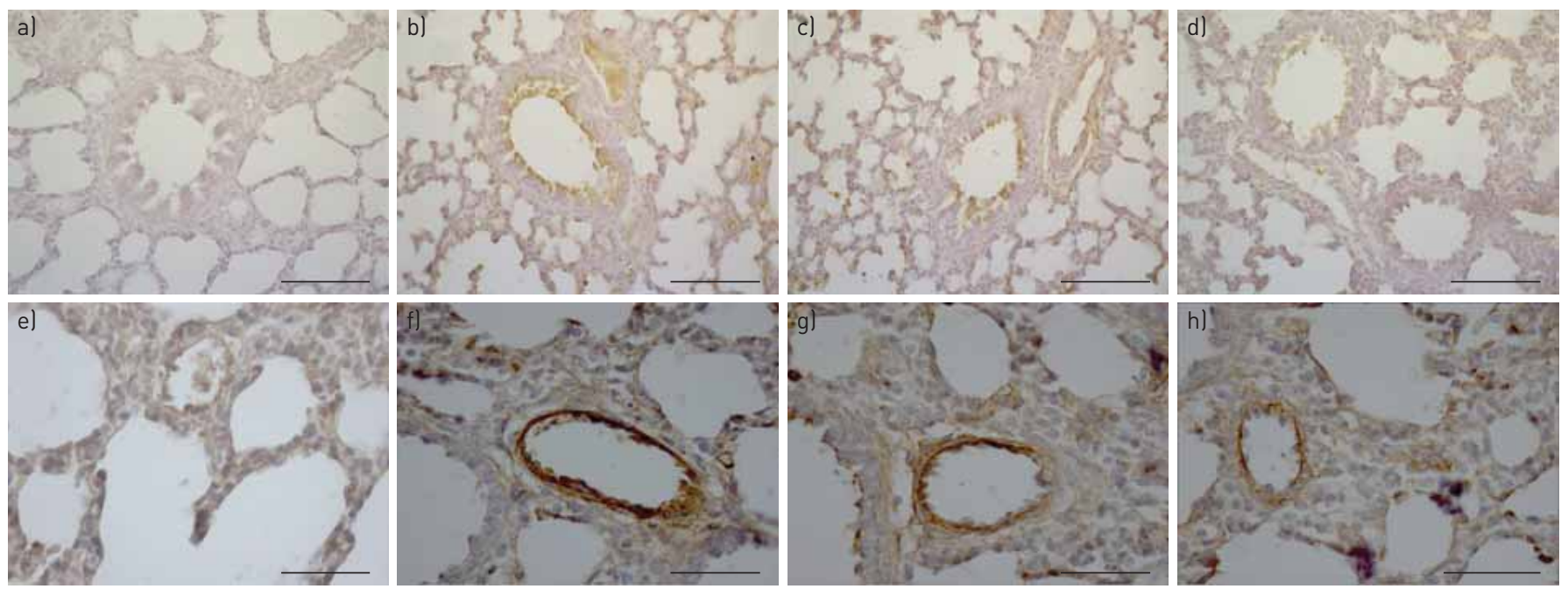

FIGURE 2 Representative images of endothelial nitric oxide synthase (eNOS) immunohistochemically stained in paraffin-embedded lung sections at post-natal day 7: a-d) bronchial structures and e-h) arterial structures. a, e) Negative control (primary antibody was replaced by mouse IgG serum at the same dilution). b, f) Rat pups kept in room air (control group). c, g) Rat pups kept in a Plexiglas chamber continuously filled with NO at 5 ppm (iN0-5 ppm group). d, h) Rat pups kept in a Plexiglas chamber continuously filled with NO at 20 ppm (iNO-20 ppm group). Scale bar: $50 \mu \mathrm{m}$.

decrease of eNOS expression in the iNO-20 ppm group, by $20 \%$ in arterial sections $(\mathrm{p}<0.01)$ and $44 \%$ in bronchial sections $(\mathrm{p}<0.001)$, compared with the control group. However, there was no significant difference in eNOS protein between the iNO-5 ppm and control groups in arterial and bronchial sections ( $p>0.05$ for both cases). At P14, pulmonary eNOS expression was not different between the three groups of rats ( $\mathrm{p}=0.55$ in arterial structures and $\mathrm{p}=0.23$ in bronchial structures) (figure $3 \mathrm{~b}$ ).

\section{Decreased eNOS mRNA synthesis in lungs from iNO-20 ppm rat pups}

Quantitative RT-PCR was used to quantify the amounts of eNOS mRNA in rat lungs in order to determine whether decreased protein expression of eNOS following iNO treatment was at the transcriptional level or not. At P7, pulmonary levels of eNOS mRNA were significantly different between the three groups of rats $(p<0.0001)$. The amount of lung eNOS mRNA in the iNO-20 ppm group was lower than in the control group ( $45 \%$ of the control group, $\mathrm{p}<0.05$ ). There was no difference in eNOS mRNA amounts between the iNO-5 ppm and control groups (figure 4a). At P14, the eNOS mRNA levels were comparable between the three groups of rat pups $(\mathrm{p}=0.45)$ (figure $4 \mathrm{~b})$.
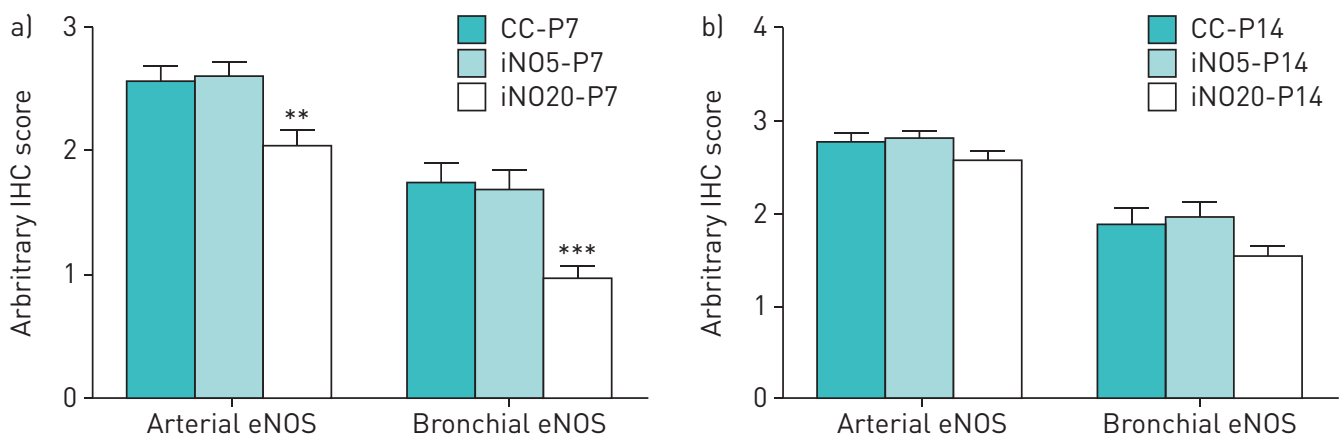

FIGURE 3 Expression of endothelial nitric oxide synthase (eNOS) evaluated by immunostaining in arterial and bronchial sections at a) post-natal day (P) 7 and b) P14. For semiquantitative analyses of eNOS expression, an arbitrary immunohistochemistry $(\mathrm{IHC})$ intensity scoring system was predetermined and evaluated by two observers: no staining (0), focal staining (1), diffuse weak staining (2), diffuse moderate staining (3) and diffuse strong staining (4). CC: rat pups kept in room air (control group); iN05: rat pups kept in a Plexiglas chamber continuously filled with $\mathrm{NO}$ at $5 \mathrm{ppm}$ (iNO-5 ppm group); iNO20: rat pups kept in a Plexiglas chamber continuously filled with $\mathrm{NO}$ at $20 \mathrm{ppm}$ (iNO-20 ppm group). a) At P7, eNOS expression was significantly lower in the iNO-20 ppm group than in the control group in arterial $(p<0.01)$ and bronchial $(p<0.005)$ sections. b) At $\mathrm{P} 14$, there was no significant difference of eNOS expression among the three groups of rats $(p>0.05)$. **: $p<0.01 ;{ }^{* * *}: p<0.005$ versus the control group (CC-P7). 
Imbalance of NOS and SOD activities in the lungs from rat pups treated with iNO

The activity of NOS and SOD in lung homogenates was assessed in all groups of animals. Whilst the activity of NOS decreased significantly in the lungs of rats in the iNO-20 ppm group to 55\% of that of the control group at P7 $(\mathrm{p}<0.01)$, the activity of SOD increased significantly $(\mathrm{p}<0.01)$ in the iNO-5 ppm group to $160 \%$ and in the iNO-20 ppm group to $185 \%$ of that of the control group at P7 (figure 5). There was no significant difference in NOS or SOD activity among the three groups of rats at P14 ( $\mathrm{p}=0.79$ and $\mathrm{p}=0.25$, respectively) (data not shown).

\section{Discussion}

Recent reports showed that the rate of iNO utilisation in the treatment of pre-term neonates continues to increase in the USA [17] with no established evidence of the beneficial effects of iNO on survival in babies without bronchopulmonary dysplasia $[3,4,18]$. In this context, understanding the side-effects of iNO, including the rebound pulmonary hypertension upon iNO withdrawal, could be useful. The results of our study showed that treatment with iNO at $20 \mathrm{ppm}$ decreased not only the activity of NOS, but also the expression of eNOS in bronchial and arterial sections. Attenuation of eNOS protein expression was at least partially due to inhibition of gene transcription. Furthermore, the activity of SOD was increased in lungs of rats treated with iNO. The effects of iNO on eNOS expression and NOS/SOD activities were reversible 1 week after withdrawal of iNO (P14).

iNO is a selective pulmonary vasodilator since it does not affect systemic vascular resistance [19]. The rebound pulmonary hypertension phenomenon was first reported in two small series of children suffering from post-operatory pulmonary hypertension, treated with iNO. Mean pulmonary arterial pressure rose significantly after iNO weaning by $54 \%$ and $64 \%$, respectively $[5,6]$. The rebound pulmonary hypertension dimmed uneventfully within $60 \mathrm{~min}$ [6]. MiLler et al. [5] suggested this phenomenon was related to downregulation of endogenous NO production. Further studies actually found a significant decrease in NOS activity during iNO treatment (between 20 and $40 \mathrm{ppm}$ ) in animal models that endured briefly after NO weaning [7-13]. The reduction of endogenous NO production coupled with discontinuation of exogenous NO supply leads to decreased NO availability, responsible for a re-augmentation of pulmonary arterial pressure and resistance during rebound pulmonary hypertension [5].

Previous studies demonstrated that NO exposure inhibited eNOS activity but not gene expression [7, 8]. In animal and/or endothelial cell models, iNO stimulated xanthine oxidase [7] and activated the ET-1/ET-A receptor pathway [9-11], leading to superoxide production and peroxynitrite formation that inactivates eNOS protein by $S$-nitration. The use of an ET-A antagonist or superoxide scavenger can prevent rebound pulmonary hypertension in animal models after acute iNO withdrawal [9-12]. Increased plasma levels of ET-1 during iNO treatment and delayed reduction of its concentration upon iNO discontinuation were also observed in patients with pulmonary hypertension or acute respiratory distress syndrome [20]. In the present study, total NOS activity decreased in the lungs of rat pups treated with $20 \mathrm{ppm}$ iNO at P7 and NOS activity reduction was completely reversible 1 week after iNO withdrawal (figure 5). Whilst our
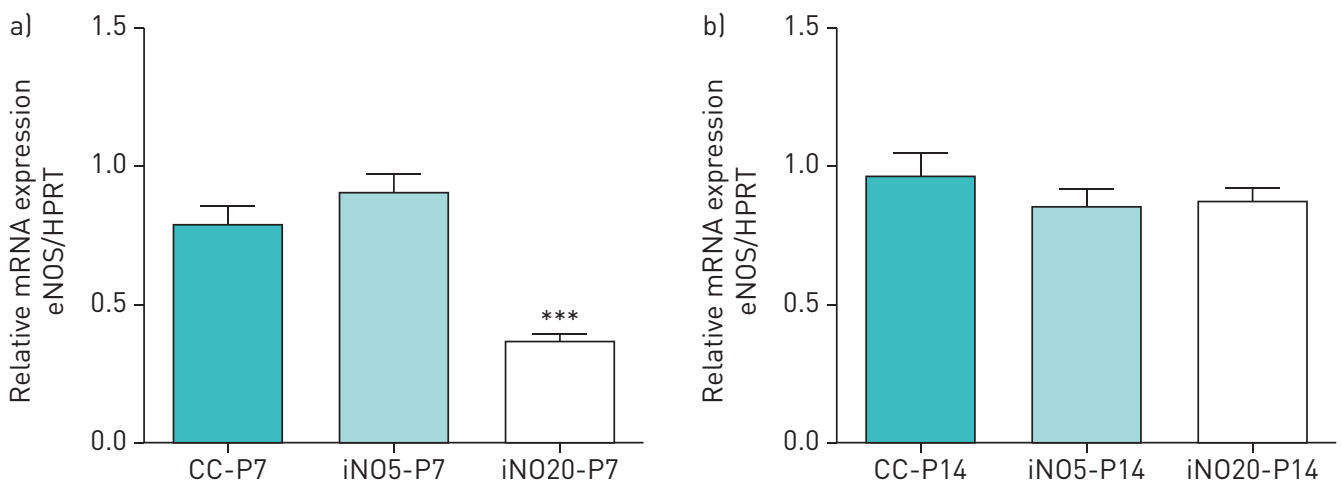

FIGURE 4 Quantification of endothelial nitric oxide synthase (eNOS) mRNA by quantitative reverse transcriptase (RT-PCR) in whole-lung homogenates at a) post-natal day (P) 7 and b) P14. Relative gene expression of eNOS in rat lungs was assessed by quantitative RT-PCR. Results were normalised to the amounts of hypoxanthine-guanine phosphoribosyltransferase (HPRT) mRNA. CC: rat pups kept in room air (control group); iNO5: rat pups kept in a Plexiglas chamber continuously filled with NO at $5 \mathrm{ppm}$ (iNO-5 ppm group); iNO20: rat pups kept in a Plexiglas chamber continuously filled with NO at $20 \mathrm{ppm}$ (iNO-20 ppm groupl. a) At P7, the amount of lung eNOS mRNA in the iNO-20 ppm group was significantly decreased as compared with that in the control group $(p<0.005)$. b) At P14, the amounts of lung eNOS mRNA were comparable among the three groups of rat $(p>0.05) .{ }^{* * *}$ : $p<0.005$ versus the control group (CC-P7). 


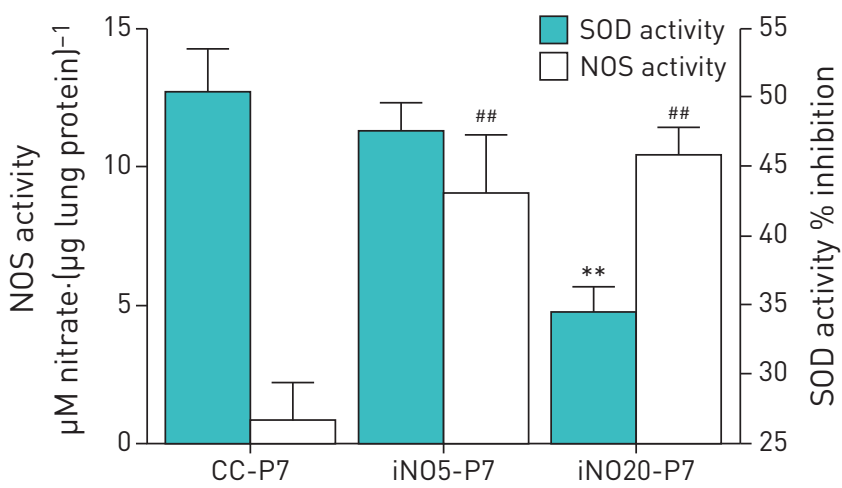

FIGURE 5 Measurement of nitric oxide synthase (NOS) and superoxide dismutase (SOD) activities in rat lung homogenates at post-natal day 7 (P7). CC: rat pups kept in room air (control group); iNO5: rat pups kept in a Plexiglas chamber continuously filled with $\mathrm{NO}$ at $5 \mathrm{ppm}$ (iNO-5 ppm group); iNO20: rat pups kept in a Plexiglas chamber continuously filled with $\mathrm{NO}$ at $20 \mathrm{ppm}$ (iNO-20 ppm group). Total NOS activity was indirectly assessed by determining the concentration of nitrate in rat lung homogenates ( $\mu \mathrm{M}$ nitrate. ( $\mu$ g lung tissue protein) ${ }^{-1}$ ) and data are presented as mean \pm SEM. The activity of SOD was measured by a colorimetric method and data are presented as the percentage inhibition of formation of formazan as described in the Methods section. The activity of NOS was significantly decreased in the lungs of the iNO-20 ppm group versus the control group $(p<0.01)$, whilst the activity of SOD was significantly increased in the iNO-5 ppm and iNO-20 ppm groups ( $p<0.01$ for both cases) as compared with that of the control group. ${ }^{* *}$ : $p<0.01$ versus the control group for NOS activity; $\#$ : $p<0.01$ versus the control group for SOD activity.

findings are consistent with other studies [7-12], they also indicate that the inhibitory effects of iNO, administered for a short period of time (i.e. 1 week, P7), are transient, and that eNOS expression and activity are restored 1 week after iNO withdrawal (P14). In addition, our results showed an increase of SOD activity in the lungs of rat pups treated with iNO (figure 5). The increased SOD activity is in accordance with our previous results that iNO treatment even at the high dose of $20 \mathrm{ppm}$ did not enhance oxidative stress as evaluated by 3-nitrotyrosine expression [2]. In fact, in some pathological conditions such as cigarette smoke exposure and acute lung injury, constitutive NOS activity is affected and inducible NOS is stimulated, leading to increased reactive oxygen species formation, and the SOD enzymes are nitrated and inactivated. SOD activity is decreased under these conditions, favouring increased superoxide and aggravated oxidative stress, forming a vicious circle [21].

Previous studies investigating the effects of iNO on eNOS expression gave different results [7-13]. Experiments on lambs with a fetal aorto-pulmonary vascular graft (shunt lambs) showed that total NOS activity was not decreased, but lung tissue eNOS protein levels during iNO (40 ppm) therapy were reduced by $43 \%$ of the baseline level [13]. In addition, expression of the $\beta$-subunit of soluble guanylate cyclase was inhibited, whilst that of phosphodiesterase type 5 was unchanged. Withdrawal of iNO was accompanied with an acute increase in pulmonary vascular resistance by $45 \%$ of the baseline value and a decrease in cyclic guanosine monophosphate (cGMP) concentrations as compared with the baseline level. Taken together, these findings suggest a profound alteration of the NO/cGMP pathway following iNO therapy [13]. Phosphodiesterase type 5 inhibitors (e.g. dipyridamole or sildenafil) might be a promising therapeutic strategy for prevention of rebound pulmonary hypertension during iNO treatment [22-24]. Another report showed that in vitro exposure of human umbilical vein endothelial cells to NO donors significantly decreased eNOS expression at the protein and gene levels as well as total NOS activity [25]. Decreased endogenous NO production upon prolonged exposure to exogenous NO might account for a blunted long-term benefit of NO treatment for patients with cardiovascular risk factors [25]. These findings were similar to our results showing a decrease in in vivo lung eNOS expression at the protein (figure 1) and gene (figure 4) levels, and more precisely in endothelial and epithelial sections (figures 2 and 3) following iNO administration. Moreover, our results also evidenced for the first time that a low dose of iNO (5 ppm) did not have any effect on eNOS expression and activity.

The limitations of the present study are the lack of assessment of hemodynamic changes and the NO/cGMP pathway upon eNOS, such as soluble guanylate cyclase and phosphodiesterase expression and activity.

\section{Conclusions}

Our results have demonstrated that eNOS expression and activity are transiently affected in newborn rat lungs during iNO treatment, linking the inhibitory effects of iNO on endogenous NO production and the rebound pulmonary hypertension phenomenon usually observed in neonatal intensive care units. 


\section{References}

1 Kumar P, Committee on Fetus and Newborn. Use of inhaled nitric oxide in preterm infants. Pediatrics 2014; 133: $164-170$.

2 Duong-Quy S, Hua-Huy T, Pham H, et al. Early inhaled nitric oxide at high dose enhances rat lung development after birth. Nitric Oxide 2014; 38: 8-16.

3 Mercier JC, Hummler H, Durrmeyer X, et al. Inhaled nitric oxide for prevention of bronchopulmonary dysplasia in premature babies (EUNO): a randomised controlled trial. Lancet 2010; 376: 346-354.

4 Kinsella JP, Cutter GR, Steinhorn RH, et al. Noninvasive inhaled nitric oxide does not prevent bronchopulmonary dysplasia in premature newborns. J Pediatr 2014; 165: 1104-1108.

5 Miller OI, Tang SF, Keech A, et al. Rebound pulmonary hypertension on withdrawal from inhaled nitric oxide. Lancet 1995; 346: 51-52.

6 Atz AM, Adatia I, Wessel DL. Rebound pulmonary hypertension after inhalation of nitric oxide. Ann Thorac Surg 1996; 62: 1759-1764.

7 Sheehy AM, Burson MA, Black SM. Nitric oxide exposure inhibits endothelial NOS activity but not gene expression: a role for superoxide. Am J Physiol 1998; 274: L833-L841.

8 Black SM, Heidersbach RS, McMullan DM, et al. Inhaled nitric oxide inhibits NOS activity in lambs: potential mechanism for rebound pulmonary hypertension. Am J Physiol 1999; 277: H1849-H1856.

9 McMullan DM, Bekker JM, Johengen MJ, et al. Inhaled nitric oxide-induced rebound pulmonary hypertension: role for endothelin-1. Am J Physiol Heart Circ Physiol 2001; 280: H777-H785.

10 Chen L, He H, Fernandez Mondejar E, et al. Endothelin-1 and nitric oxide synthase in short rebound reaction to short exposure to inhaled nitric oxide. Am J Physiol Heart Circ Physiol 2001; 281: H124-H131.

11 Wedgwood S, McMullan DM, Bekker JM, et al. Role for endothelin-1-induced superoxide and peroxynitrite production in rebound pulmonary hypertension associated with inhaled nitric oxide therapy. Circ Res 2001; 89: 357-364.

12 Oishi P, Grobe A, Benavidez E, et al. Inhaled nitric oxide induced NOS inhibition and rebound pulmonary hypertension: a role for superoxide and peroxynitrite in the intact lamb. Am J Physiol Lung Cell Mol Physiol 2006; 290: L359-L366.

13 Ross GA, Oishi P, Azakie A, et al. Endothelial alterations during inhaled NO in lambs with pulmonary hypertension: implications for rebound hypertension. Am J Physiol Lung Cell Mol Physiol 2005; 288 : L27-L35.

14 Pham H, Vottier G, Pansiot J, et al. Inhaled NO protects cerebral white matter in neonatal rats with combined brain and lung injury. Am J Respir Crit Care Med 2012; 185: 897-899.

15 Bei Y, Duong-Quy S, Hua-Huy T, et al. Activation of RhoA/Rho-kinase pathway accounts for pulmonary endothelial dysfunction in patients with chronic obstructive pulmonary disease. Physiol Rep 2013; 1 : e00105.

16 Pham H, Vottier G, Pansiot J, et al. Inhaled NO prevents hyperoxia-induced white matter damage in neonatal rats. Exp Neurol 2014; 252: 114-123.

17 Ellsworth MA, Harris MN, Carey WA, et al. Off-label use of inhaled nitric oxide after release of NIH consensus statement. Pediatrics 2015; 135: 643-648.

18 Finer NN, Evans N. Inhaled nitric oxide for the preterm infant: evidence versus practice. Pediatrics 2015; 135 754-756.

19 Hataishi R, Zapol WM, Bloch KD, et al. Inhaled nitric oxide does not reduce systemic vascular resistance in mice. Am J Physiol Heart Circ Physiol 2006; 290: H1826-H1829.

20 Pearl JM, Nelson DP, Raake JL, et al. Inhaled nitric oxide increases endothelin-1 levels: a potential cause of rebound pulmonary hypertension. Crit Care Med 2002; 30: 89-93.

21 Salvemini D, Doyle TM, Cuzzocrea S. Superoxide, peroxynitrite and oxidative/nitrative stress in inflammation. Biochem Soc Trans 2006; 34: 965-970.

22 Ivy DD, Kinsella JP, Ziegler JW, et al. Dipyridamole attenuates rebound pulmonary hypertension after inhaled nitric oxide withdrawal in postoperative congenital heart disease. J Thorac Cardiovasc Surg 1998; 115: 875-882.

23 Thelitz S, Bekker JM, Ovadia B, et al. Inhaled nitric oxide decreases pulmonary soluble guanylate cyclase protein levels in 1-month-old lambs. J Thorac Cardiovasc Surg 2004; 127: 1285-1292.

24 Namachivayam P, Theilen U, Butt WW, et al. Sildenafil prevents rebound pulmonary hypertension after withdrawal of nitric oxide in children. Am J Respir Crit Care Med 2006; 174: 1042-1047.

25 Schmidt A, Bilgasem S, Lorkowski S, et al. Exogenous nitric oxide regulates activity and synthesis of vascular endothelial nitric oxide synthase. Eur J Clin Invest 2008; 38: 476-485. 